УДК 159.9

DOI 10.25205/2658-4506-2019-12-1-115-132

\author{
Е. А. Дорошева \\ Новосибирский государственный университет \\ Ул. Пирогова, 1, Новосибирск, 630090, Россия \\ Elena.dorosheva@mail.ru

\section{МОДЕЛИ ФОРМИРОВАНИЯ РАС: ВОЗМОЖЕН ЛИ ДИАЛОГ? *}

\begin{abstract}
Рассматривается проблема возможности диалога ряда подходов к формированию расстройств аутистического спектра. Сравниваются особенности методических основ в подходах, основывающихся на модели психического; подходах, основанных на межличностных взаимодействиях; подходах, разработанных в рамках модели сенсорной интеграции. В дополнение к ранее выделенным «сквозным» феноменам, связанным с развитием РАС (интолерантность к определенности и дефицитарность самовосприятия), описывается вклад нарушений межличностной привязанности. Делается вывод о возможности интеграции подходов путем рассмотрения базовых феноменов в развитии РАС.

Ключевые слова: расстройства аутистического спектра, дети с РАС, модель психического, сенсорная интеграция, отношение к ситуациям неопределенности, самовосприятие, межличностная привязанность.
\end{abstract}

Расстройства аутистического спектра (далее - РАС) относятся к дизонтогенетическим нарушениям, возникают на ранних этапах развития детей, проявляются на разных уровнях (от молекулярного до поведенческого) и характеризуются тяжелым течением. В настоящее время число диагностированных случаев РАС быстро растет.

\footnotetext{
* Исследование поддержано грантом РФФИ (проект № 18-013-00925).
}

Дорошева Е. А. Модели формирования РАC: возможен ли диалог? // Reflexio. 2019. T. 12, № 1. С. 115-132.

Reflexio. 2019. Tом 12, № 1

(C) E. А. Дорошева, 2019 
Существует множество подходов, итоговой целью которых является абилитация детей с РАС (именно ранние вмешательства позволяют получить больше позитивных изменений). Ключевым вопросом в рамках каждого подхода (помимо прикладного анализа, работающего с поведенческими проявления синдрома) выступают предполагаемые причины возникновения РАС, модели их развития

Богатый материал, наработанный в рамках каждого из подходов, зачастую оказывается изолированным в его границах, поскольку используются собственная терминология, специфическая логика построения системы причинно-следственных связей. Одной из важных проблем остается оценка эффективности предлагаемых методов, которая оказывается затруднена принципиальной разницей подходов, разрабатываемых в рамках разных моделей РАС.

Нужно отметить, что появляется все больше метааналитических работ, направленных как на анализ исследований, осуществленных в рамках одного подхода, так и на сравнение результатов, полученных при применении разных подходов. Авторы указывают, что сравнения, особенно во втором случае, существенно затрудняются разницей критериев, выбираемых разными исследователями (см., например, мета-анализ исследований, в которых оценивалась эффективность коррекционных программ трех направлений - [Tachibana et al., 2017]).

Задача сравнения разных подходов оказывается особенно актуальной, поскольку в работе с конкретным ребенком речь может идти об индивидуальном подборе подхода или о сочетании разных подходов. Важность метаанализа также связана с тем, что единичные экспериментальные исследования охватывают небольшие выборки, в них входят дети с очень разными нарушениями (как правило, контролируется только важнейший фактор уровня интеллектуального развития), что связано с самой спецификой РАС. Более того, появляются обзоры метаанализов, сравнивающих сами метааналитические подходы и выявляющие, как особенности их проведения повлияли на выводы исследователей [Reichow, 2012]). Сравнение результатов многих исследований поможет сделать действительно обоснованные выводы, однако это требует единообразия измерений, что, в свою очередь, может основываться только на выделении «сквозных» феноменов, объединяющих различные подходы.

Цель данной работы - теоретический анализ ряда основных моделей развития РАС, с целью описания возможности выделения общих оснований, способствующих диалогу между представителями различных подходов. 


\section{Модель психического (Theory of Mind, ToM)}

В качестве одного из подходов к развитию РАС можно упомянуть представления о ключевой причине РАС, состоящей в нарушениях модели психического («Theory of Mind»). Под моделью психического понимается гипотетическая концептуальная система, лежащая в основе способности понимать (отслеживать) и объяснять свои ментальные состояния (что мы знаем, думаем, хотим, чувствуем) и ментальные состояния других людей [Baron-Cohen et al., 1985]. Наличие модели психического позволяет отделять представления других людей от реальных условий (которым они могут не соответствовать) и от собственных представлений.

Существенное поражение именно сфер коммуникации, социальных взаимодействий, воображения (в частности, возможности моделировать социальные ситуации в своих представлениях) у страдающих РАС побудило к рассмотрению нарушений (отсутствия, дефицитарности) модели психического как ключевой причины наблюдаемых нарушений [Ibid.]. Эта точка зрения подкрепляется многочисленными свидетельствами нейрофизиологов, описывающих изменения в работе default mode network - системы мозга, отвечающей за процессы саморефлексии и понимание убеждений, намерений, эмоциональных состояний других; связанной с внутренним диалогом (см. обзор: [Padmanabhan et al., 2017]). Умственная отсталость, диагностируемая у большей части детей, страдающих РАC, не объясняет всех нарушений, наблюдаемых в социальных взаимодействиях. Специфические коммуникационные проблемы отмечаются и у детей с РАС, имеющих нормальный уровень интеллектуальных способностей, в то же время многие дети с отставанием в умственном развитии демонстрируют нормальную для общего уровня своего развития социальную компетентность [Сергиенко и др., 2009].

Таким образом, предполагается, что существующее в психике образование - модель психического - отвечает за спектр связанных поведенческих проявлений, делает их возможным. Выявление дефицитарности модели психического у детей с РАС, а также оценка эффективности воздействий, направленных на ее восполнение, предполагает выявление способностей, возникающих на основе модели психического. Помимо оценки ответа на задание также может рассматриваться вопрос: «Почему респондент дал именно такой ответ?», поскольку основным моментом является интерпретация психических процессов, стоящих за поведенческой реакцией. 
Ключевой момент нарушения при РАС с точки зрения данной теории - это именно невозможность понимать других (mind reading). То, что лежит в основе этой неспособности, по-разному понимается различными авторами - предложен ряд подходов к развитию и структуре модели психического. Как база для ее развития рассматривается объединенное с другими внимание (само по себе или как часть общего внимания), врожденные способности к имитации и к восприятию аффективных стимулов и социальным реакциям и т. д. [Сергиенко и др., 2009; Baron-Cohen et al., 2000; Hobson, 1993]. Однако акцент делается именно на совокупности взаимосвязанных способностей. Также в данном подходе ставятся вопросы о возможном влиянии на развитие модели психического общих когнитивных способностей (либо рассмотрении их как основы для ее формирования) и проводится сравнение развития моделей ментального и физического мира. Обсуждается проблема: имеет ли смысл при оценке развития детей с РАС для практических задач коррекции проверять уровень понимания физических законов (которое также нарушено, но в меньшей степени, чем понимание законов социального мира) или имеет смысл исследовать лишь сферу представлений о себе и о других?

В любом случае, акцентируются именно проблемы самопредставления и понимания других людей.

В целом, выделяют главные способности, нарушения которых описываются для детей с РАС, на двух предполагаемых уровнях модели психического и в области представлений о себе.

Первый уровень (based level Theory of Mind) - базовые (в том числе, предполагаемые врожденные, иногда выделяемые в отдельный, предварительный уровень) представления о ментальном мире:

- распознавание эмоций по лицевой экспрессии;

- понимание эмоций в контексте ситуации;

- понимание желаний и намерений других людей;

- способность отслеживать направление взгляда;

- способность к имитации.

Второй уровень (advanced level Theory of Mind), формирующийся на основе первого:

- понимание ментальных причин эмоции, ментальной причинности поведения;

- понимание психических состояний и причин второго и далее порядков (что думает другой человек о том, что думает / чувствует третий человек и т.д.); 
- прогнозирование поведения других людей на основе знаний о их желаниях и убеждениях;

- понимание отличия причин движения физических (внешний импульс) и социальных (внутреннее намерение) объектов;

- понимание обмана, умение обманывать, разделение обмана «во благо» и «во зло»;

- понимание юмора, иронии.

- Самопредставления:

- понимание зеркального я;

- наличие связных, непрерывных во времени и пространстве, телесного и психического образов себя.

Другая классификация предполагает выделение аффективных и когнитивных составляющих модели психического [Baron-Cohen et al., 2000]. За каждой психической способностью, выявляемой в рамках модели, могут стоять одновременно когнитивные и аффективные составляющие (например, описывают эмоциональные и когнитивные компоненты эмпатии [Perry, Shamay-Tsoory, 2013]).

Разработан ряд задач, которые позволяют прямо (например, называние эмоциональных состояний, объяснение поведения персонажа в предлагаемой истории и др.) или косвенно (характер действий по отношению к своему отражению в зеркале и т.д.) оценить сформированность данных способностей (см., например: [Сергиенко и др., 2009]). Проявления данных способностей за рамками эксперимента, в повседневной жизни измеряют вербальные опросники для родителей, учителей и видеозаписи социальных взаимодействий и эмоциональной экспрессии [Golan, Baron-Cohen, 2006]. (Отмечается, что используются эти методы измерения неоправданно редко [Begeer et al., 2010]).

Проводимые абилитационные методы воздействия основаны на тренировке отдельных способностей модели психического (разных уровней - например, имитация, распознавание эмоций, понимание осведомленности других, понимание ошибочных знаний других, понимание сложного юмора и т.д.) [Begeer et al., 2011].

\section{Сенсорная и моторная интеграция}

Теория о недостаточной сенсорной интеграции как ключевой причине развития симптомов РАС проистекает из идеи, впервые высказанной Дж. Айресом [Ayres, 1991]. Она заключается в том, что у людей, страдающих РАС, наблюдается дефицит в модулях моз- 
га, отвечающих за процессы ощущений и восприятия, в результате чего мозг не справляется с обработкой сенсорных сигналов и созданием целостных образов. Это приводит к нарушению когнитивных процессов, основывающихся на восприятии, нарушению целостности поведения, а также вызывает перегрузку - резко снижает устойчивость к воздействию сенсорных стимулов одной или разных модальностей, к влиянию социальных стимулов; вызывает защитные реакции - предпочтения привычной обстановки, действий и т. д.

Цель абилитационных воздействий в рамках данного подхода улучшить сенсорную модуляцию, связанную с поведением и вниманием, и посредством этого повысить способности к социальному взаимодействию, когнитивные навыки, возможности саморегуляции. Применяемые техники ориентированы на то, чтобы помочь нервной системе модулировать, организовывать и интегрировать информацию из окружающей среды; также они направлены на интеграцию моторной сферы, вторично страдающей в процессе развития. В результате улучшения сенсорной интеграции реакции на внешние стимулы становятся более адаптивными [Baranek, 2002]. Кроме того, подразумевается, что повышение сенсорной интеграции ведет к повышению стрессоустойчивости (увеличивается переносимость стимулов разной интенсивности, более адаптивным становится поведение в новых ситуациях и т.д.).

В рамках данного подхода разработан ряд вербальных опросников, с помощью которых эксперты-наблюдатели, родители или учителя могут оценить показатели сенсорной и моторной интеграции. Например, Sensory Processing Measure (7 шкал - показатели работы зрительной, слуховой, тактильной, проприорицептивной, вестибулярной, моторной систем и социального восприятия), Quick Neurological Screening Test (15 шкал, в частности, оценка целенаправленных моторных действий, ловкости, зрительного отслеживания, пространственной ориентации, тактильных способностей, двигательных навыков, восприятия) и др. [Pfeiffer et al., 2013]. По-видимому, в комплексных исследованиях они могут служить органичным дополнением прочих методов.

\section{Подходы, основанные на межличностных взаимодействиях}

Ряд подходов рассматривает РАС, отталкиваясь от ключевой идеи нарушения коммуникации. Предлагаемые в них практические абилитационные воздействия основаны, в основной или существенной 
части, на вовлечении ребенка в коммуникацию. Чаще как базовая рассматривается коммуникация со взрослыми (прежде всего - родителями, также - учителями).

Необходимо отметить, что идея нарушений коммуникации как ключевых при РАС пересекается с подходами модели психического (в частности, могут включаться в обзоры литературы, посвященные модели психического), однако если тренинги в данном подходе направлены на развитие ряда умений модели психического, то подходы, концентрирующиеся на межличностных взаимодействиях, отталкиваются от идеи контакта, который естественным образом стимулирует и направляет ребенка.

Одно из предположений в рамках данных подходов основывается на том, что ключевыми для развития РАС выступают нарушения в эмоциональной сфере. Такой точки зрения придерживался Р. П. Хобсон, утверждая, что дети, страдающие РАС, имеют врожденную неспособность воспринимать эмоции других людей и отвечать на них [Hobson, 1993]. Он предлагал стимулировать развитие у детей общего социального опыта, рассказывая о событиях, касающихся ребенка, показывая его фотографии, и демонстрируя эмоциональную окрашенность воспоминаний. Е. Р. Баенская, О. С. Никольская описывают совместно-разделенные переживания и предлагают технологию разделения аффективных переживаний с ребенком для стимуляции развития его аффективной и коммуникативной сфер [2014]. Авторы также указывают на постоянную необходимость в наполнении взрослым происходящего вокруг смыслами (обсуждение мыслей, чувств других людей, причин, подтекстов ситуаций и т. д.).

Близкое содержание у модели DIR (Developmental Individualdifference Relationship-based model), предполагающей выстраивание близких взаимоотношений с ребенком, основывающихся на понимании его индивидуальных особенностях, погружении в его мир и затем, когда сформировавший доверие ребенок сам этого захочет, вовлечении его в реальный мир [Wieder, 2013].

Еще одна модель данной группы концентрируется на трудностях детей с РАС в области «объединенного внимания» - присоединения ко взрослому в направлении внимания на какой-либо предмет или событие. Отмечается, что дети с РАС не понимают указательных жестов руки, направления взгляда, и некоторые исследователи полагают, что именно эти нарушения лежат в основе широкого спектра нарушений социального поведения. Метод формирования объединенного внимания предполагает стимуляцию концентрации внимания 
взрослого и ребенка на каком-либо предмете / событии, вовлечении ребенка в этот процесс; таким образом, создается трехстороннее взаимодействие и формируется как предметная, так и коммуникативная деятельность ребенка (см., например: [Bondy, Frost, 1995]).

Так же как ключевые рассматриваются трудности имитации. У детей и взрослых с РАС отмечается нарушение способности имитации лицевой экспрессии, которая, при нормативном развитии, характерна уже для новорожденных. Вслед за нарушениями имитации эмоциональных выражений лица проявляются трудности «эмоционального заражения» - присоединения к эмоциям окружающих. Предполагается, что в дальнейшем это может вести к нарушению восприятия другого как «похожего на себя» [Meltyff, Gopnik, 1993]. Открытие зеркальных нейронов, составляющих нейрофизиологическую основу имитации, и описание их дефицитарности у людей с РАС дополнительно привлекло внимание к идеям о центральной роли нарушений имитации в формировании РАС [Якобони, 2011]. Направленное развитие имитации также становится центральным моментом в ряде абилитационных подходов.

Итак, в рамках данных подходов установление контакта не только необходимый этап для того, чтобы ребенок смог обучаться, но сам контакт рассматривается как побуждение к развитию. Ребенок не выполняет / не только выполняет специально подобранные задания, но контактирующий человек присоединяется к нему, либо привлекает его внимание и действия к тому, чем занимается сам, вовлекает в переживания, дает пример осмысливания происходящего и вовлекает в обсуждение смыслов, и т. д.

Как метод коррекционного воздействия рассматривается и вовлечение ребенка в коммуникацию с другими детьми. Это, например, подход К. Хигаши - терапия повседневной жизнью. В основе его лежит установление тесных связей между ребенком с РАС и его близкими, обучение в коллективе сверстников, создание интенсивной совместной деятельности, в которой обучение должно происходить от других детей через синхронизацию (установление ритмов жизни) и имитацию [Quill et al., 1989]. Для достижения этих целей формируются группы, включающие только детей с РАС, где основная функция организация контактов принадлежит психологу (см., например: [Власова, 2014]), группы, в которых поддерживающая функция в значительной степени принадлежит сверстникам с нормативным развитием [Kohler et al., 1995]. Последняя задача зачастую решается в рамках развития инклюзивного обучения детей с РАС. 
Нужно отметить, что идея стимуляции контакта рассматривается не только с целью восполнения дефицитарности модели психического, но и как способствующая усилению центральной интеграции, которая будет рассмотрена далее (другой человек помогает ребенку «воедино» собирать то, что для ребенка фрагментарно). Центральная интеграция, в свою очередь, помогает формированию саморегуляции и способностейк планированию своих действий.

Особым феноменом, рассматриваемым в данной группе моделей, выступает межличностная привязанность - установление тесных эмоциональных связей с близкими людьми. Привязанность у детей и взрослых с РАС имеет многочисленные нарушения. В некоторых случаях она может не формироваться, т. е. дети не проходят нормативный этап разделения на «своих» и «чужих». Другое типичное нарушение состоит в том, что дети, напротив, чрезмерно требуют постоянного присутствия матери, категорически не хотят оставаться с другими людьми. Сформировавшаяся и определяемая как надежная привязанность у детей с РАС отличается от классического описания этого типа привязанности: дети беспокоятся, если мать уходит, но в ее присутствии не вовлекают ее в игровую деятельность, меньше обращаются к ней, не контактируют глазами. Тем не менее надежная привязанность у детей с РАС отмечается достаточно часто, хотя и реже, чем у нормативно развивающихся детей [Koren-Karie et al., 2009; Oppenheim et al., 2009]. Формирование надежной привязанности у детей с РАС осложняется не только их особенностями, но и сложностями с положительной обратной связью для родителей, высоким уровнем стресса, который отмечается у родителей, что негативно сказывается на отношении родителей к ребенку. Отмечается, что родительский стресс существенно воздействует на состояние ребенка [Tachibana et al., 2017]. Ряд авторов считают, что надежный тип привязанности не связан с тяжестью симптомов РАС, и, по-видимому, его формирование не ведет к облегчению патологической симптоматики, однако он явно способствует адаптации ребенка в социальной среде [Dolev et al., 2014].

Нужно отметить, что при нормативном становлении привязанности этапы ее формирования тесно связаны с возможностью отличения близких людей и соответствуют этапам перцептивного развития ребенка. Например, нормативный этап разделения «своих» и «чужих» в 6-8 месяцев жизни ребенка связывают с созреванием константности зрительного восприятия - образ матери становится стабилен при любом бытовом изменении ее внешности [Newcombe, 1996]. Мы можем 
предполагать, что нарушения сенсорной интеграции могут быть тесно связаны с изменениями, отмечаемыми для привязанности у детей c PAC.

Огромный вклад в формирование того или иного типа привязанности детей вносит поведение родителей по отношению к детям. Показано, что матери, которые сенситивны к потребностям ребенка, т. е. понимают их и дают адекватный ответ, существенно способствуют развитию надежной привязанности [Koren-Karie et al., 2009]. Также рассматривается такое свойство, как проницательность матерей само их желание понять мотивы, причины поведения ребенка, его чувства и мысли, индивидуальные особенности. Показано, что проницательность матерей также тесно связана с формированием у ребенка надежной привязанности [Oppenheim et al., 2009]. Это свойство более доступно измерению, поскольку сенситивность предполагает наличие информации о потребностях ребенка, чтобы проверить их совпадение с представлениями и действиями матери, а это достаточно сложно для экспертной оценки. Проницательность легче поддается измерению [Ibid.].

Изучение типа привязанности у детей с РАС проводится с помощью классического эксперимента «Незнакомая ситуация», применяемого для детей 2-6 лет [Koren-Karie et al., 2009; Oppenheim et al., 2009]. По всей видимости, информативными также могут быть и видеозаписи поведения ребенка в обыденной жизни, при контакте с близкими и незнакомыми людьми. Также возможна разработка опросников для родителей, диагностирующих тип привязанности ребенка; опросники могут дополнять экспериментальные данные. Проницательность и сенситивность матерей измеряются, в частности, с помощью постэкспериментального интервью, предполагающего обсуждение видеофрагментов взаимодействия матери и ребенка [Oppenheim et al., 2009].

Представляется целесообразным рассмотрение РАС в контексте семейного взаимодействия. Ход психического и психофизиологического развития ребенка, его самочувствие, поведенческие проявления тесно связаны со средой развития, которой в первую очередь является семья. В значительном числе работ, включая наши исследования, отмечаются существенные изменения в самочувствии и поведении родителей, связанные с наличием в семье ребенка, страдающего РАС [Дорошева, Грабельникова, 2018]. Таким образом, учет общего семейного контекста может внести существенный вклад в понимание динамики РАС. 


\section{Возможности выделения феноменов, лежащих в основе развития РАC, общих для рассмотренных моделей}

Таким образом, при анализе трех групп подходов выявляется наличие объединяющих оснований. Ранее были описаны такие общие феномены, определяющие РАС, как снижение толерантности к ситуациям неопределенности (проявление базовой дисфункциональной схемы обработки информации) [Первушина, 2018] и нарушения самовосприятия [Первушина, Хорошилов, 2018]. Оба феномена четко прослеживаются в трех рассмотренных группах моделей. Дефицитарность модели психического приводит, прежде всего, к дезориентации в ситуациях социального взаимодействия, увеличению уровня их неопределенности, что, как следствие, может вызывать многочисленные нарушения, отмечаемые феноменологически в коммуникативных моделях РАС. Нарушения сенсорной интеграции ведут к разобщенности, фрагментарности общей картины мира, что увеличивает неопределенность самого широкого круга ситуаций. Нарушения самовосприятия связаны с недостаточностью модели психического, что приводит к дефицитарности понимания других, отделению их от себя, обозначению своих границ, самопонимания через других. Это также приводит к широкому спектру нарушений коммуникации. Недостаточность сенсорной интеграции приводит к нарушениям основ самовосприятия, разрушению телесного «Я», на базе которого формируется «Я» психическое.

Как еще один «сквозной» феномен мы можем выявить привязанность к значимым людям. Привязанность, формируясь в раннем возрасте, лежит в основе межличностных контактов, и ее нарушения могут быть связаны с широким спектром нарушений способностей, описываемых моделью психического, и проявляться в специфике коммуникации, описываемой соответствующей группой моделей. Становление привязанности связано с созреванием перцептивных процессов, и ее нарушения могут быть тесно связаны с дефицитом сенсорной интеграции. Мы считаем, что привязанность должна изучаться в более широком контексте семейных взаимодействий, в том числе, совместно с изучением характеристик матерей, влияющих на ее формирование (сенситивность, привязанность).

Анализ общих феноменов, связанных с развитием РАС, может быть основанием для диалога, взаимообогащения и, наконец, интеграции различных моделей развития РАС, в том числе, в области их 
выхода на практическую работу с детьми, страдающими РАС. Такая интеграция выглядит весьма перспективной.

Рассмотренные нами исследовательские методы, предлагаемые в рамках разных подходов, могут быть использованы совместно, в том случае, если целью исследовательской деятельности становится изучение общих феноменов, характеризующих развитие РАС.

\section{Список литературы}

Власова Л. И. Коммуникативные игры на занятиях по подготовке к школе детей с расстройствами аутистического спектра // Аутизм и нарушения развития. 2014. № 4 (45). С. 42-46.

Дорошева Е. А., Грабельникова У. С. Синдром эмоционального выгорания, особенности совладающего поведения и регуляции эмоций у матерей, воспитывающих ребенка с расстройством аутистического спектра // Reflexio. 2018. T. 11, №1. C. 5-18.

Никольская О. С., Баенская Е. Р. Коррекция детского аутизма как нарушения аффективной сферы: содержание подхода // Альманах Института коррекционной педагогики. 2014. № 19. URL: https://alldef. ru/ru/articles/almanah-19/korrekcija-detskogo-autizma-kak-narushenija (дата обращения 03.09.2018).

Первушина О. Н. Аутизм через призму отношения к неопределенности // Восьмая международная конференция по когнитивной науке. Светлогорск, 2018. С. 803-805.

Первушина О. Н., Хорошилов Б. М. О роли самовосприятия в когнитивном и социальном функционировании аутистов // Современные проблемы клинической психологии и психологии личности. Материалы Всерос. науч.-практ. конф. Новосибирск, 2018. С. 104-108.

Сергиенко Е. А., Лебедева Е. И., Прусакова О. А. Модель психического как основа становления себя и другого в онтогенезе человека. М.: Изд-во Ин-та психологии РАН, 2009.

Якобони М. Отражаясь в людях: Почему мы понимаем друг друга. М.: ООО «Юнайтед Пресс», 2011.

Ayres A. J. Sensory integration and learning disorders. Los Angeles: Western Psychological Services, 1991.

Baranek G. T. Efficacy of sensory and motor interventions for children with autism // Journal of Autism and Developmental Disorders. 2002. Vol. 32. P. 397-422.

Baron-Cohen S., Leslie A. M., Frith U. Does the autistic child have a "theory of mind"? Cognition, 1985. Vol. 21. P. 37-46. 
Baron-Cohen S., Tager-Flusberg H., Cohen D. J. Understanding other minds: Perspectives from developmental cognitive neuroscience. Oxford: Oxford Uni. Press, 2000.

Begeer S., Malle B. F., Nieuwland M. S., Keysar B. Using Theory of Mind to represent and take part in social interactions: Comparing individuals with high-functioning autism and typically developing controls // European Journal of Developmental Psychology. 2010. Vol. 7. P. 104122.

Begeer S., Gevers C., Clifford P., Verhoeve M., Kat K., Hoddenbach E., Boer F. Theory of Mind Training in Children with Autism: A Randomized Controlled Trial // Journal of Autism and Developmental Disorders, 2011. Vol. 41. P. 997-1006.

Bondy A. S., Frost L. A. Educational approaches in preschool: Behavior techniques in a public school setting. In: E. Schopler et al. (eds.) Learning and cognition in autism. New York: Plenum Press, 1995. P. 311-333.

Dolev S., Oppenheim D., Koren-Karie N., Yirmiya N. Early attachment and maternal insightfulness predict educational placement of children with autism // Research in Autism Spectrum Disorders. 2014. Vol. 8. P. 958-967.

Golan O., Baron-Cohen S. Systemizing empathy: Teaching adults with Asperger syndrome or high-functioning autism to recognize complex emotions using interactive multimedia // Development and Psychopathology, 2006. Vol. 18. P. 591-617.

Hobson R. P. Autism and the Development of Mind. Hillsdale: Laurence Erlbaum Association, 1993.

Kohler F. W., Strain P. S., Hoyson M., Davis L., Donina W. M., Rapp $N$. Using a Group-Oriented Contingency to Increase Social Interactions between Children with Autism and Their Peers // A Preliminary Analysis of Corollary Supportive Behaviors. 1995. Vol. 19, No.1. P. 10-32.

Koren-Karie N., Oppenheim D., Dolev S., Yirmiya N. Mothers of securely attached children with autism spectrum disorder are more sensitive than mothers of insecurely attached children // Journal of Child Psychology and Psychiatry, 2009. Vol. 50, No. 5. P. 643-650.

Meltyff A.N., Gopnik A. The role of imitation in understanding persons and developing a theory of mind. In: S. Baron-Cohen, H. Tager-Flusberg and D. Cohen (eds.). Understanding other minds: perspectives from autism. Oxford: Oxford Uni. Press. Google Scholar, 1993. P. 335-366.

Newcombe N. Child development: Change over time. New York: HarperCollins, 1996.

Oppenheim D., Koren-Karie N., Dolev S., Yirmiya N. Maternal insightfulness and resolution of the diagnosis are associated with secure 
attachment in preschoolers with autism spectrum disorders // Child Development. 2009. Vol. 80, No. 2. P. 519-527.

Padmanabhan A., Lynch C. J., Schaer M., Menon V. The Default Mode Network in Autism. Biological Psychiatry: Cognitive Neuroscience and Neuroimaging, 2017. Vol. 2. P. 476-486.

Perry A., Shamay-Tsoory S. Understanding emotional and cognitive empathy:neuropsychological perspective. In: S. Baron-Cohen, H. TagerFlusberg, M. V. Lombordo (eds.). Understanding other minds. Perspectives from developmental social neuroscience. Oxford: Oxford Uni. Press, 2013. P. 178-195.

Pfeiffer B. A., Koenig K., Kinnealey M., Sheppard M., Henderson L. Effectiveness of Sensory Integration Interventions in Children With Autism Spectrum Disorders: A Pilot Study // American Journal of Occupational Therapy. 2013. Vol. 65, No. 1. P. 76-85.

Reichow B. Overview of Meta-Analyses on Early Intensive Behavioral Intervention for Young Children with Autism Spectrum Disorders // Journal of Autism and Developmental Disorders, 2012. Vol. 42, № 4. P. 512-520.

Tachibana Y., Miyazaki C., Ota E., Hwang Y., Kobayashi E., Terasaka A., Tang J., Quill K., Gurry S., Larkin A. Daily Life Therapy: A Japanese model for educating children with autism // Journal of Autism and Developmental Disorders. 1989. Vol. 19, No. 4. P. 625-635.

Tachibana Y., Miyazaki C., Ota E., Mori R., Hwang Y., Kobayashi E., Terasaka A., Tang J., Kamio Y. A sistematic rewiew and meta-analysis of comprehensive interventions for pre-school children with autism spectrum disorder (ASD) // PLoS One, 2017. Vol. 6, № 12 :e0186502. Available in: https:/www.ncbi.nlm.nih.gov/pubmed/29211740 (12.04.2019).

Wieder S. Developmental, Individual Difference, Relationship-Based (DIR) Model In: Volkmar F. R. (ed.). Encyclopedia of Autism Spectrum Disorders. New York: Springer, 2013. 


\author{
E. A. Dorosheva \\ Novosibirsk State University \\ 1 Pirogov Str., Novosibirsk, 630090, Russian Federation \\ Elena.dorosheva@mail.ru
}

\title{
THE MODELS DESCRIBING ASD FORMATION: WHETHER DIALOGUE IS POSSIBLE?
}

Whether dialogue is possible between different models describing formation autism spectrum disorders (ASD)? This problem is considered. Features of methodical bases and the corresponding terminology in the model of mind, approaches based on interpersonal interactions, model of sensory integration were compared. In addition to earlier described "through" phenomena connected with development of RAS (intolerance to uncertainty and deficiency of self-perception) the contribution of interpersonal attachment disturbances was described. The conclusion about ability to integrate approaches by consideration of basic phenomena in development of RAS is drawn.

Keywords: autism spectrum disorders (ASD), children with ASD, theory of mind, sensory integration, tolerance to uncertainty, self-perception, interpersonal attachment.

\section{References}

Vlasova L. I. (2014). Kommunikativnye igry na zanyatiiah po podgotovke k shkole detej s rasstrojstvami autisticheskogo spectra [Communicative games in the classroom to prepare for school children with autism spectrum disorders]. Autizm i narusheniya razvitiia, 4 (45), 42-46 (in Russ.)

Grinspen S. (2015). Na ty s autizmom. Ispol'zovanie metodiki floortime [Engaging Autism. Using the Floortime Approach to Help Children Relate, Communicate, and Think]. Moskva: Terevinf, 2015 (in Russ.)

Dorosheva E. A., \& Grabelnikova U. K. (2018) Sindrom jemocional'nogo vygoranija, osobennosti sovladajushhego povedenija i reguljacii jemocij u materej, vospityvajushhih rebenka s rasstrojstvom autisticheskogo spektra [Emotional burnout, specific of coping strategies and emotional 
regulation in the mothers of children with authism spectrum disorders]. Reflexio, 11 (1), 5-18 (in Russ.)

Nikol'skaya O. S., \& Baenskaya E. R. (2014). Korrekciia detskogo autizma kak narusheniia affektivnoj sfery: soderzhanie podhoda [Correction of children's autism as a disturbance of the affective sphere: the content of the approach]. Al'manah Instituta korrekcionnoj pedagogiki, 19. Retrieved from: https://alldef.ru/ru/articles/almanah-19/korrekcijadetskogo-autizma-kak-narushenija (03.09.2018) (in Russ.)

Pervushina O. N., Khoroshilov B. M. (2018) Autizm cherez prizmu otnoshenija $\mathrm{k}$ neopredelennosti [Authism through a prism of the attitude towards uncertainty]. Vos'maja mezhdunarodnaja konferencija po kognitivnoj nauke [The eighth international conference on cognitive science]. Svetlogorsk, 803-805 (in Russ.)

Pervushina O. N., Khoroshilov B. M. (2018). Sovremennye problemy klinicheskoj psihologii i psihologii lichnosti [About the role of selfperception in the cognitive and social functioning of authism]. Materialy Vserossijskoj nauchno-prakticheskoj konferencii. [Modern problems of clinical psychology and psychology of the personality. Materials of the All-Russian scientific and practical conference]. Novosibirsk, 104108 (in Russ.)

Sergienko E. A., Lebedeva E. I., \& Prusakova O. A. (2009). Model' psihicheskogo kak osnova stanovleniia sebia i drugogo $\mathrm{v}$ ontogeneze cheloveka [The Theory of Mind as the basis for the emergence of self and others in the human ontogenesis]. M.: Izdatel'stvo Instituta psihologii RAN (in Russ.)

Iacoboni M. (2011). Otrazhaias'v liudiah: Pochemu my ponimaem drug druga [Mirroring People: The New Science of How We Connect with Others]. M.: OOO "Iunajted Press". (in Russ.)

Ayres A. J. (1991). Sensory integration and learning disorders. Los Angeles: Western Psychological Services.

Baranek G. T. (2002). Efficacy of sensory and motor interventions for children with autism. Journal of Autism and Developmental Disorders, 32, 397-422.

Baron-Cohen S., Leslie A. M., \& Frith U. (1985). Does the autistic child have a "theory of mind"? Cognition, 21, 37-46.

Baron-Cohen S., Tager-Flusberg H., \& Cohen D. J. (2000). Understanding other minds: Perspectives from developmental cognitive neuroscience. Oxford: Oxford Uni. Press.

Begeer S., Malle B. F., Nieuwland M. S., \& Keysar B. (2010). Using Theory of Mind to represent and take part in social interactions: Comparing 
individuals with high-functioning autism and typically developing controls. European Journal of Developmental Psychology, 7, 104-122.

Begeer S., Gevers C., Clifford P., Verhoeve M., Kat K., Hoddenbach E., \& Boer F. (2011). Theory of Mind Training in Children with Autism: A Randomized Controlled Trial. Journal of Autism and Developmental Disorders, 41, 997-1006.

Bondy A. S., \& Frost L. A. (1995). Educational approaches in preschool: Behavior techniques in a public school setting. In: E. Schopler et al. (eds.), Learning and cognition in autism. New York: Plenum Press, pp. 311-333.

Dolev S., Oppenheim D., Koren-Karie N., \& Yirmiya N. (2014). Early attachment and maternal insightfulness predict educational placement of children with autism. Research in Autism Spectrum Disorders, 8, 958967.

Golan O., \& Baron-Cohen S. (2006). Systemizing empathy: Teaching adults with Asperger syndrome or high-functioning autism to recognize complex emotions using interactive multimedia. Development and Psychopathology, 18, 591-617.

Hobson R. P. (1993). Autism and the Development of Mind. Hillsdale: Laurence Erlbaum Association.

Kohler F.W., Strain P.S., Hoyson M., Davis L., Donina W.M., \& Rapp N. (1995). Using a Group-Oriented Contingency to Increase Social Interactions between Children with Autism and Their Peers. A Preliminary Analysis of Corollary Supportive Behaviors, 19 (1), 10-32.

Koren-Karie N., Oppenheim D., Dolev S., \& Yirmiya N. (2009). Mothers of securely attached children with autism spectrum disorder are more sensitive than mothers of insecurely attached children. Journal of Child Psychology and Psychiatry, 50, (5), 643-650.

MeltyffA. N., \& GopnikA. (1993). The role of imitation in understanding persons and developing a theory of mind. In: S. Baron-Cohen, H. TagerFlusberg, \& D. Cohen (eds), Understanding other minds: perspectives from autism. Oxford: Oxford University Press. Google Scholar, 335-366.

Newcombe N. (1996). Child development: Change over time. New York: HarperCollins.

Oppenheim D., Koren-Karie N., Dolev S, \& Yirmiya N. (2009). Maternal insightfulness and resolution of the diagnosis are associated with secure attachment in preschoolers with autism spectrum disorders. Child Development, 80 (2), 519-527.

Padmanabhan A., Lynch C. J., Schaer M., \& Menon V. (2017). The Default Mode Network in Autism. Biological Psychiatry: Cognitive Neuroscience and Neuroimaging, 2, 476-486. 
Perry A., \& Shamay-Tsoory S. (2013). Understanding emotional and cognitive empathy:neuropsychological perspective. In: S. Baron-Cohen, H. Tager-Flusberg, \& M.V. Lombordo (eds.). Understanding other minds. Perspectives from developmental social neuroscience. Oxford: Oxford Uni. Press, 178-195.

Pfeiffer B. A., Koenig K., Kinnealey M., Sheppard M., \& Henderson L. (2013). Effectiveness of Sensory Integration Interventions in Children With Autism Spectrum Disorders: A Pilot Study. American Journal of Occupational Therapy, 65 (1), 76-85.

Reichow B. (2012). Overview of Meta-Analyses on Early Intensive Behavioral Intervention for Young Children with Autism Spectrum Disorders. Journal of Autism and Developmental Disorders, 42 (4), 512-520.

Tachibana Y., Miyazaki C., Ota E., Hwang Y., Kobayashi E., Terasaka A., Tang J., Quill K., Gurry S., \& Larkin A. (1989). Daily Life Therapy: A Japanese model for educating children with autism. Journal of Autism and Developmental Disorders, 19 (4), 625-635.

Tachibana Y., Miyazaki C., Ota E., Mori R., Hwang Y., Kobayashi E., Terasaka A., Tang J., Kamio Y. (2017) A sistematic rewiew and metaanalysis of comprehensive interventions for pre-school children with autism spectrum disorder (ASD) PLoS One, 2017, 6(12) :e0186502. Available in: https://www.ncbi.nlm.nih.gov/pubmed/29211740 (12.04.2019).

Wieder S. (2013) Developmental, Individual Difference, Relationship-Based (DIR) Model. In: Volkmar F. R. (ed.), Encyclopedia of Autism Spectrum Disorders. New York: Springer. 